RUECTRONIC FILE AVAMASLA

Preprint

UCRL-JC- 135024

\title{
Monte Carlo simulations of arterial imaging with optical coherence tomography
}

\author{
P. Amendt, K. Estabrook, M. Everett, R.A. London, \\ D. Maitland, G. Zimmerman, B. Colston, L. da Silva, \\ U. Sathyam
}

This article was submitted to SPIE/BIOS Conference on Gastrointestinal Surgery, San Jose, CA, January 22, 2000

U.S. Department of Energy

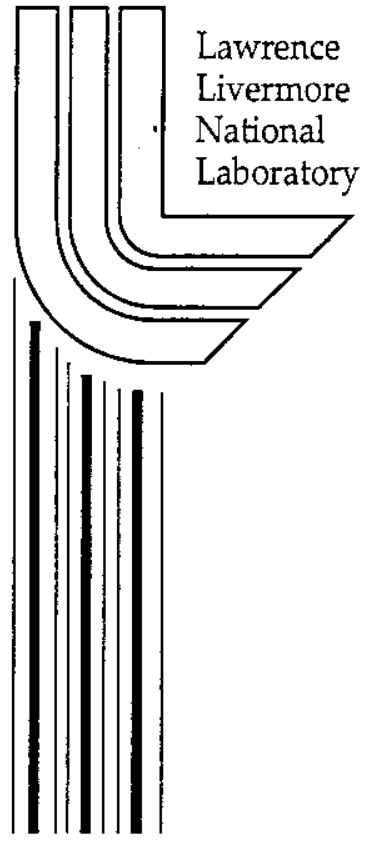

February 1, 2000

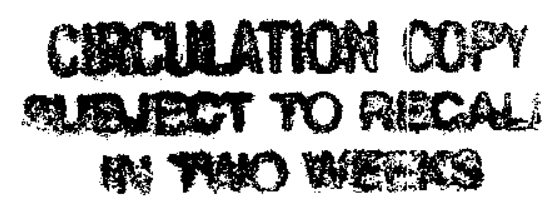

Approved for public release; further dissemination unlimited 


\section{DISCLAIMER}

This document was prepared as an account of work sponsored by an agency of the United States Government. Neither the United States Government nor the University of California nor any of their employees, makes any warranty, express or implied, or assumes any legal liability or responsibility for the accuracy, completeness, or usefulness of any information, apparatus, product, or process disclosed, or represents that its use would not infringe privately owned rights. Reference herein to any specific commercial product, process, or service by trade name, trademark, manufacturer, or otherwise, does not necessarily constitute or imply its endorsement, recommendation, or favoring by the United States Government or the University of California. The views and opinions of authors expressed herein do not necessarily state or reflect those of the United States Government or the University of California, and shall not be used for advertising or product endorsement purposes.

This is a preprint of a paper intended for publication in a journal or proceedings. Since changes may be made before publication, this preprint is made available with the understanding that it will not be cited or reproduced without the permission of the author.

This report has been reproduced directly from the best available copy.

Available to DOE and DOE contractors from the Office of Scientific and Technical Information

P.O. Box 62, Oak Ridge, TN 37831

Prices available from (423) 576-8401

http://apollo.osti.gov/bridge/

Available to the public from the

National Technical Information Service

U.S. Department of Commerce 5285 Port Royal Rd., Springfield, VA 22161

http://www.ntis.gov/

\section{OR}

Lawrence Livermore National Laboratory

Technical Information Department's Digital Library

http://www.llnl.gov/tid/Library.html 


\title{
Monte Carlo simulations of arterial imaging with optical coherence tomography
}

\author{
Peter Amendt, K. Estabrook, M. Everett, R.A. London, D. Maitland, G. Zimmerman, B. Colston, \\ L. da Silva and U. Sathyam ${ }^{\dagger}$
}

Lawrence Livermore National Laboratory, University of California. CA 94550

\begin{abstract}
The laser-tissue interaction code LATIS [London et al., Appl. Optics 36, 9068 (1998)] is used to analyze photon scattering histories representative of optical coberence tomography (OCT) experiments performed at Lawrence Livermore National Laboratory. Monte Carlo photonics with Henyey-Greenstein anisotropic scattering is implemented and used to simulate signal discrimination of intravascular structure. An analytic model is developed and used to obtain a scaling law relation for optimization of the OCT signal and to validate Monte Carlo photonics. The appropriateness of the Henyey-Greenstein phase function is studied by direct comparison with more detailed Mie scattering theory using an ensemble of spherical dielectric scatterers. Modest differences are found between the two prescriptions for describing photon angular scattering in tissue. In particular, the Mie scattering phase functions provide less overall reflectance signal but more signal contrast compared to the Henyey-Greenstein formulation.
\end{abstract}

Keywords: Optical coherence tomography, arterial imaging, Mie scattering, endoscopy, Henyey-Greenstein

\section{Introduction}

Because of its high spatial resolution and noncontact nature, optical coherence tomography (OCT) is gaining attention as a potentially useful intravascular imaging diagnostic.' The 10-20 micron range in resolution of OCT provides at least an order of magnitude improvement over ultrasound or other tomographic techniques, e.g., computed tomography and magnetic resonance imaging.

OCT imaging works by superposing a reference beam and a probe beam of the tissue sample to produce interference fringes when the path length difference is within a coherence length of the light source [See Fig. (1)]. A broadband source is sought in order to reduce the coberence length, thereby providing the

a)

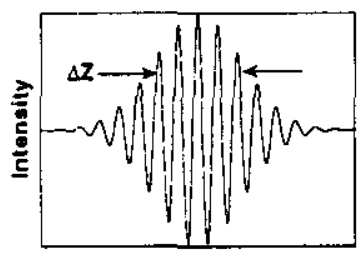

Pathiength miamatch b)

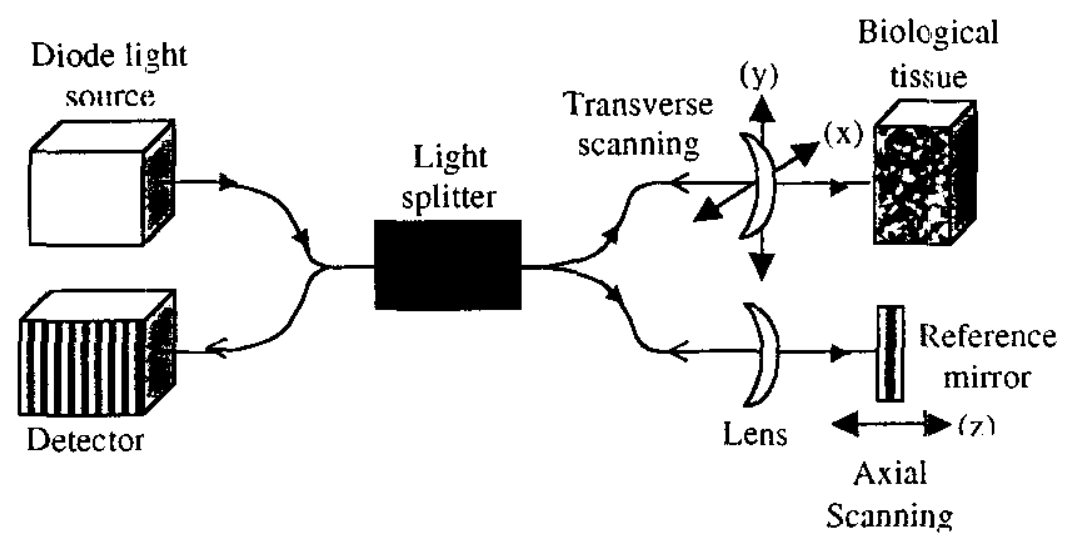

Fig. 1a-b: a) Simulated interferogram where $\Delta z$ is the coherence length of the diode light source. b) Schematic of LLNL OCT experiment 
desired high spatial resolution. For intravascular applications the medium through which the sampling photons propagate is often highly scattering or turbid. Thus, photons which backscatter from well inside the tissue into the interferometer can undergo a large pathlength increase from many large-angle scattering events. This pathlength increase may result in too few photons interfering with the reference beam to give a useful image. The challenge posed by OCT in highly scattering media is to optimize the return signal to the point that several millimeters of tissue depth can be effectively scanned.

Recently. researchers have begun to identify and understand the sources of signal degradation in OCT. Pan et al. used Monte Carlo techniques to study the path-length resolved reflectance signal in tissue phantoms. ${ }^{2}$ However, the simulated reflectance signal was not resolved into single- and multiple-scattering contributions which could shed further light on the properties of OCT. They conjectured that local inhomogeneities may give rise to speckle-related phenomena in the OCT signal. Schmitt and Knüttel have adapted an extended Huygens-Fresnel formulation of beam propagation in a turbulent atmosphere to the study of speckle in $\mathrm{OCT}^{3}$ They concluded that speckle from multiple scattering severely degrades image quality in OCT by causing large fluctuations in the measured heterodyne signal. Schmitt et al. have recently proposed several techniques for reducing speckle in OCT. ${ }^{+}$Finally, Yao and Wang have looked more closely at the role of multiple scattering in OCT and the use of polarization-sensitive OCT to experimentally indicate when multiple scattering prevails. ${ }^{5}$

In this Proceeding we consider a number of research areas of relevance to OCT. In Section II, we develop an analytic theory of signal propagation through a highly scattering medium based on the HenyeyGreenstein formulation of angular scattering. Such an exercise is useful because it provides a consistency check of the Monte Carlo calculations which are often computationally expensive. More importantly, an analytic treatment provides scaling law relations which often prove useful in identifying an optimal region of parameter space in which to operate. We present a simple example for an optimized OCT signal by adjusting the sample beam radius $a_{0}$, given a source coherence length $\Delta z$, a probe distance $z_{d}$, a HenyeyGreenstein anisotropy parameter $g$, and an isotropic scattering inverse mean-free-path $\alpha^{-1}$.

Next, Section III describes the application of the two-dimensional laser-tissue interaction code ${ }^{6}$ LATIS for understanding photon scattering phenomena germane to the LLNL OCT experiment. A Henyey-Greenstein angular scattering function is implemented in the Monte Carlo photonics package to model the high clegree of scattering anisotropy found in vascular tissue. A simulated path-length resolved reflectance signal is presented which shows qualitative agreement with experiments using porcine vascular tissue. The simulated scattering history of all detected photons which reach the vessel boundary is recorded and analyzed. We find that the simulated OCT signal is characterized by many small-angle scattering events and the notable absence of single-scattering events.

The Henyey-Greenstein formulation of angular scattering is common to current and past efforts at describing OCT with Monte Carlo photonics. In Section IV we consider the Mie theory of scattering which rigorously accounts for the interaction of radiation with matter when the dimensions of the scatterer are on the the order of the wavelength of light or greater. This investigation is worthwhile for two reasons. First, large-angle backscattering is an important component of OCT both as a source of background noise and as a requirement for detecting a signal photon from a given depth in the sample. Consequently, a more exact treatment is needed to check the validity of the Henyey-Greenstein formulation for this important class of scattering event. Second, Mie theory automatically includes polarization effects. We have recently included a Mie theory of angular scattering in the Monte Carlo package of LATIS. We find that the HenyeyGreenstein formulation represents a reasonably valid simplification of Mie theory in vascular tissue according to the cases we have studied to date. A comparison of the OCT signal level and the associated scattering history for the two formulations of angular scattering shows acceptable agreement. Finally, we compare the results of a LATIS polarization-sensitive simulation of OCT in a blood vessel with an unpolarized version; we find little difference between the two cases when the blood and vessel walls are treated as homogeneous and isotropic. 


\section{Analytical Modeling}

A useful exercise is to estimate the OCT signal from analytical modeling alone. In this way, useful consistency checks of simulation results and identification of interesting parameter regimes are possible. We pose the following problem: How much signal degradation occurs in an initially uniform beam of photons propagating parallel through a homogeneous and isotropic medium many mean-free-paths long and characterized by strong anisotropic scattering? Our analysis will be based on a Henyey-Greenstein description of angular scattering but is generalizable to other scattering functions. The Henyey-Greenstein phase function reads:

$$
f_{H G}(\theta)=\frac{l}{4 \pi} \frac{1+g^{2}}{\left[1+g^{2}-2 g \cos (\theta)\right]^{3 / 2}}
$$

where $0 \leq g<1$ is the anisotropy parameter and $\theta$ is the angle of scattering relative to the direction of the incident photon. The medium is still termed isotropic because the anisotropic angular scattering has no preferred incident direction; otherwise, the medium is anisotropic as in fibrous tissue. The HenyeyGreenstein phase function has the convenient property that

$$
\langle\cos \theta\rangle \equiv \int_{0}^{2 \pi} d \phi \int_{-1}^{1} d(\cos \theta) \cos \theta f_{H G}(\theta)=g
$$

where $\phi$ is the azimuthal angle. Given an initially uniform beam of photons, the radial profile after one mean-free-path $\left(\alpha^{-1}\right)$ of propagation can be computed using viewfactor ${ }^{7}$ methods. The differential number of photons $d N$ detected by an observer over an area $d A$ situated a distance $r$ from a planar scattering source $N^{\prime} d A^{\prime}$ with differential area $d A^{\prime}$ and oriented at an angle $\theta$ is given by the following expression:

$$
d N=\frac{N^{\prime} \cdot d A^{\prime} \cdot f_{H G} \cdot \cos \theta \cdot d A}{r^{2}}
$$

To evaluate Eq. (3), we consider the geometry shown in Fig. (2) and write for the number of photons detected per unit area at radius $R$ :

$$
N(R)=N^{\prime} \int_{0}^{a} a \cdot d a \int_{0}^{2 \pi} d \phi \cdot f_{H G}[\theta(\phi)] \cdot \frac{\cos \theta(\phi)}{r^{2}}
$$

where $N^{\prime}$ is the number of source photons per unit area, $\theta=\cos ^{-1}(r / z)$, and $r=\left(R^{2}+z^{2}+a_{0}{ }^{2}-2 a_{0} R \cos \phi\right)^{1 / 2}$.

Fig. 2: Schematic of geometry used to evaluate Eq. (3).

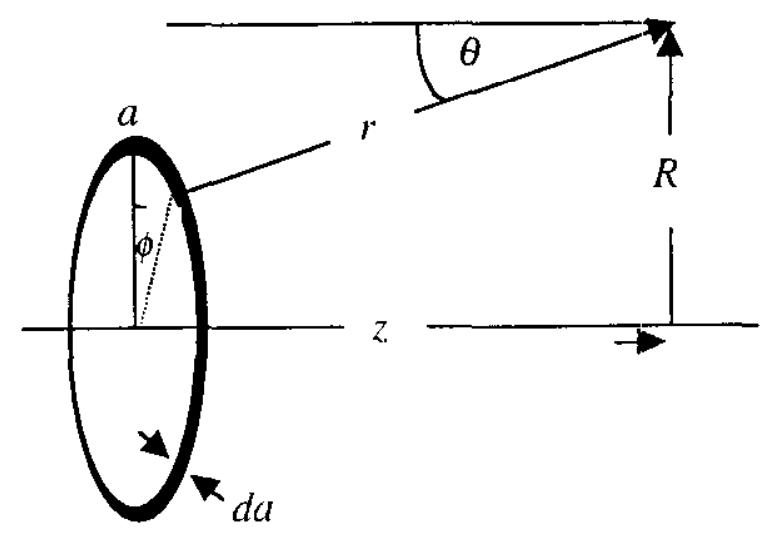



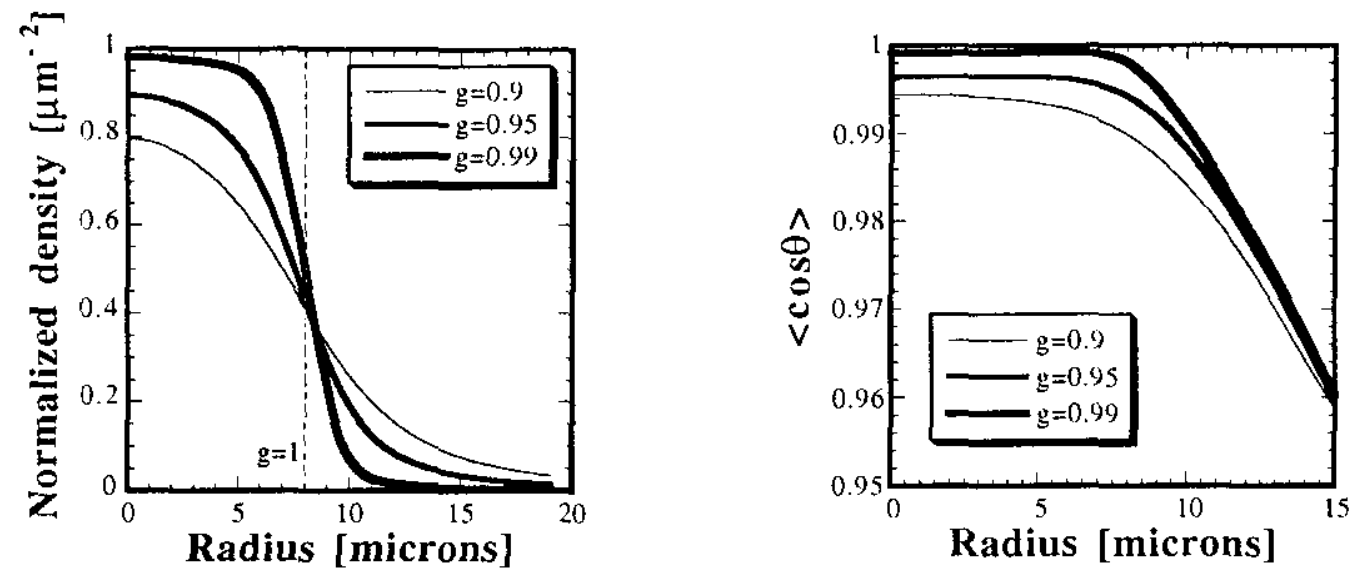

Fig. 3a, b: a) Normalized areal density of photons received versus radius $R$ for four values of HenyeyGreenstein anistropy parameter $g$ with radius of source $a_{0}=8 \mu \mathrm{m}$ and $z=\alpha^{\prime}=43 \mu \mathrm{m}$. b) Average areaweighted cosine of angle of received photons at observation radius $R$.

Figure (3a) shows the result of evaluating Eq. (4) at $z=\alpha^{-1}$ for various values of anisotropy parameter $g$. As expected, the distribution of scattered photons increases as the anisotropy parameter $g$ decreases and is accompanied by an areal density reduction on axis that scales $2(1-g)$ for $g$ close to unity. However, the extent of scattering is not as large as would be expected based on Eq. (2). For the case of $g=0.9$, $\left\langle\cos \theta=26^{\circ}\right.$ and an appreciable fraction of photons would be expected to reside outside $R=30 \mu \mathrm{m}$. Rather, the radius at which half the photons are found at $z=\alpha^{\cdot 4}$ is only about $10 \mu \mathrm{m}$. This property indicates that the appropriate angle for describing distributed scattering is not $\cos ^{-1}(g)$ but is somewhat smaller and requires another choice. Figure ( $3 b$ ) provides some indication of a possible alternative. Here we show the average of the cosine of the angle of photons received as a function of radius $R$. For sufficiently large $g$, the corresponding deflection angles are quite small and insensitive to $R<a_{0}$. This property means that the deflection angle at $R=0$ is satisfactorily representative of all deflection angles up to $R=a_{0}$. Therefore, we can analytically compute the deflection angle $\theta_{0}$ at $R=0$ and use this angle as an approximate measure of the beam emittance from the scattering plane at $z=0$. This prescription for anisotropic scattering from a distributed source should be valid provided the effect of scattering anisotropy is large compared to the effect of geometrical narrowing, i.e., $\theta_{0} \ll \tan ^{-1}\left(a_{0} \alpha\right)$.

Having proposed a choice of appropriate angle for a single scattering, we now consider a sequence of scattering events to estimate the fraction of photons still within a radius $a_{0}$ after many mean-free-paths of propagation. After the $\mathrm{i}$-th scattering event, $\theta_{0, \mathrm{i}}$ is evaluated according to the expression:

$$
\cos \left(\theta_{o, i}\right)=\frac{\int_{\mu_{o, 1-1}}^{1} \frac{d \mu}{\mu^{2}} f_{H G}(\mu, g)}{\int_{\mu_{0,-1}}^{l} \frac{d \mu}{\mu^{3}} f_{H G}(\mu, g)}
$$

where $\mu_{0,1}=1 /\left(1+a_{0, i}{ }^{2} \alpha^{2}\right)^{1 / 2}$ and $a_{0,1}$ is updated according to the prescription: $a_{0, i}=a_{0, i, 1}+\alpha^{1} \tan \left(\theta_{0, i}\right)$. After $n$ scatterings, the fraction of photons remaining within radius $a_{n}$ is then estimated as $\left(a_{1} / a_{0,0}\right)^{2}$. Figure (4) 


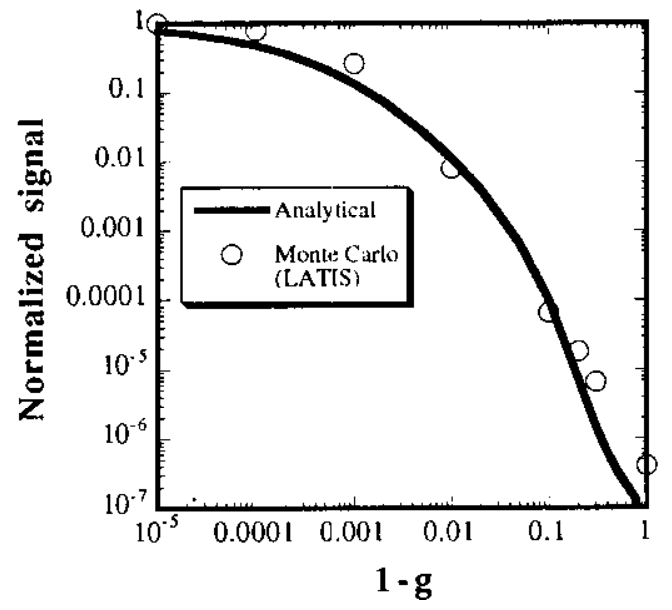

Fig. 4: Shown is the calculated detected signal normalized to the incident signal versus HG-anistropy parameter ( $1-g)$ based on analysis (solid line) and LATIS Monte Carlo simulations. The radius of the signal detection window $a_{0}=8 \mu \mathrm{m}$, the scattering length $\alpha^{1}$ is $43 \mu \mathrm{m}$, and the propagation distance $z_{d}$ is $800 \mu \mathrm{m}$.

shows the results of this procedure applied to the case described in Figs. (3a, b) but with a (round-trip) propagation distance $z_{d}=800 \mu \mathrm{m}$. Very good agreement with LATIS Monte Carlo simulations [See Section III] is found over a five-order magnitude range in anisotropy, particularly in the range of interest to vascular OCT: $\mathrm{g}=0.9$ to 0.99 .

The analysis lends itself to a study of scaling law relations which could prove useful in optimizing vascular OCT. For this purpose, we return to Eq. (5) and explicity evaluate the integrals to leading order in $(1-\mathrm{g})<<1$ to find:

$$
\cos \theta_{o} \equiv\left(1-\frac{1-a_{o}^{2} \alpha^{2} / 4}{\sqrt{1+g \cdot\left(a_{o} \alpha /(1-g)\right)^{2}}}\right) /\left(1-\frac{1-9 a_{o}^{2} \alpha^{2} / 16}{\sqrt{1+g \cdot\left(a_{o} \alpha /(1-g)\right)^{2}}}\right) .
$$

Photons which propagate a distance $z_{\mathrm{d}}$ and reach the detector radius $a_{\mathrm{o}}$ undergo a series of small angle scatterings with size on the order of $\theta_{\mathrm{o}}$. Thus, their total distance of travel is roughly given by $z_{\mathrm{d}} / \cos \theta_{\mathrm{o}}$. For optimized OCT we stipulate that the pathlength difference between these photons and unscattered signal photons be on the order of the coherence length of the source $\Delta z: z_{d}\left(1 / \cos \theta_{0}-1\right)=\Delta z$. To make further analytic progress, we limit ourselves to the case of strong anisotropy: $1-g<a_{0} \alpha$. Using this limit in Eq. (6) and applying the optimized OCT criterion, we obtain:

$$
a_{o p t} \approx \frac{\Delta z}{\alpha z_{. d}} \frac{4 \sqrt{g}}{(1-g)\left(5 / 4-\Delta z / z_{d}\right)},
$$


where $a_{o p x}$ is the optimized beam radius. Equation (7) implies that an OCT beam radius smaller than the optimum is needless since the coherence is already satisfactory. For a source coherence length of $20 \mu \mathrm{m}$. tissue depth $\alpha z_{\mathrm{d}}=19$, and tissue anisotropy $g=0.9$, Eq. (7) suggests an optimal radius is about $30 \mu \mathrm{m}$.

\section{LATIS Monte Carlo simulations: Henyey-Greenstein angular scattering}

A powerful tool for understanding OCT is the use of Monte Carlo photonic techniques applied to lasertissue interactions. ${ }^{5.8}$ We have recently adapted the two-dimensional laser-tissue interaction code ${ }^{6}$ LATIS to the study of anisotropic photon transport in isotropic tissue using Monte Carlo techniques. Figure (5) shows the geometry used in the simulations to model in vitro OCT experiments performed at LLNL.

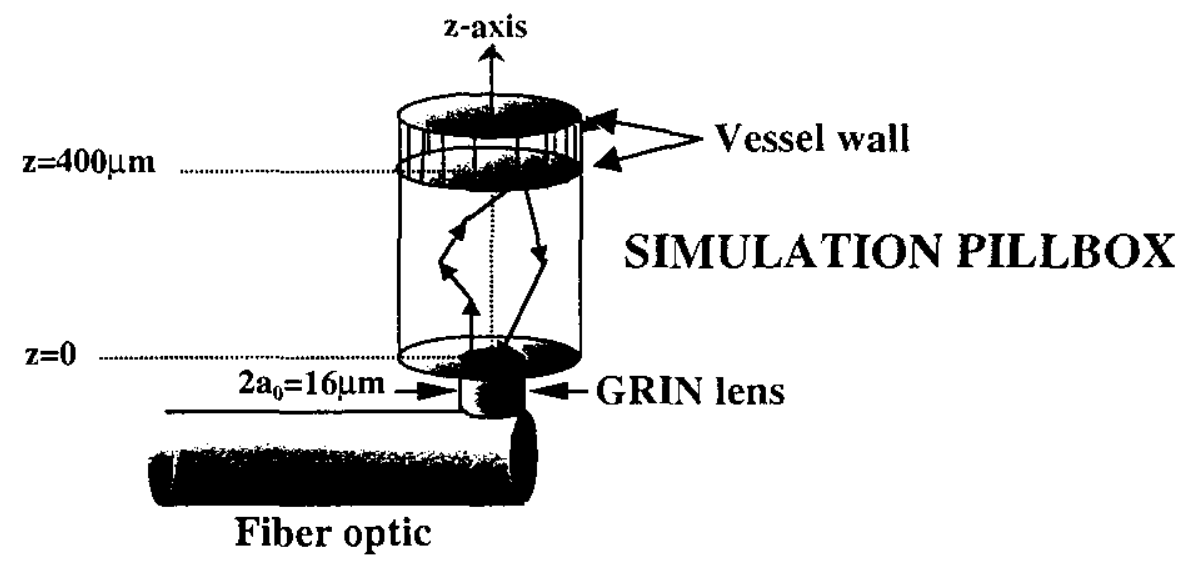

Fig. 5: Simulation geometry for LLNL OCT experiments. Monte Carlo photons (small arrows) are launched from end of fiber optic within a $2 a_{0}=16 \mu \mathrm{m}$ diameter circle at $z=0$ in direction normal to (planar) vessel wall and tracked within (cylindrically symmetric) simulation pilibox. Collected photons within same $16 \mu \mathrm{m}$ diameter circle (GRIN lens) are monitored in time to simulate OCT signal.

Monte Carlo census photons are launched each timestep at $z=0$ uniformly within a circle of radius $a_{0}$ in a direction perpendicular to the vessel wall, i.e., along the $z$-axis. Photons which propagate into the vessel wall and return to $z=0$ within $R=a_{0}$ are tagged and analyzed in terms of scattering history. The phase function assumed in the simulations is of the Henyey-Greenstein type. The light source used in the experiment operates at a central wavelength of $1.31 \mu \mathrm{m}$ with a FWHM spectral bandwidth of $47 \mathrm{~nm}$. The absorption coefficient $\mu$ of blood at this wavelength is only about $0.4 \mathrm{~cm}^{-1}$ and we take $\alpha$ of about $233 \mathrm{~cm}^{-1}$ as before for the isotropic scattering coefficient. A suite of calculations was performed first for the case of photons propagating through $400 \mu \mathrm{m}$ of blood, specularly reflecting from the vessel boundary and then returning to $z=0$ to find how many photons returned within a radius $R=a_{0}$ as $g$ was varied. Figure (4) shows the strong dependence on $g$ and the good agreement with analytical modeling as noted earlier in Section II. Figure (6a) shows the scattering history of detected photons propagating a total distance of $800 \mu \mathrm{m}$ through blood for three values of $\mathrm{g}$. In all three cases, the majority of photons returning to the detector have undergone on the order of $z_{\mathrm{d}} \alpha$ scattering events. This trend is strictly true for the strongly anisotropic case $(g=0.999)$, but becomes relaxed somewhat as the anisotropy decreases. For decreasing $g$, the average angle of scattering $\theta_{0}$ increases so that the relative likelihood of a minimally scattered photon reaching the detector must increase. Indeed, we see that the single-scatter signal strength for the case $g=0.99$ is stronger than for $g=0.999$. Overall, Fig. (6a) shows that the scattering distribution widens in a relative sense and shifts slightly to fewer scatterings as the arisotropy decreases. In Fig. (6b) we plot the detected signal versus number of scatterings for three delay times and for the case: $g=0.99$. Note that the earliest delay time 

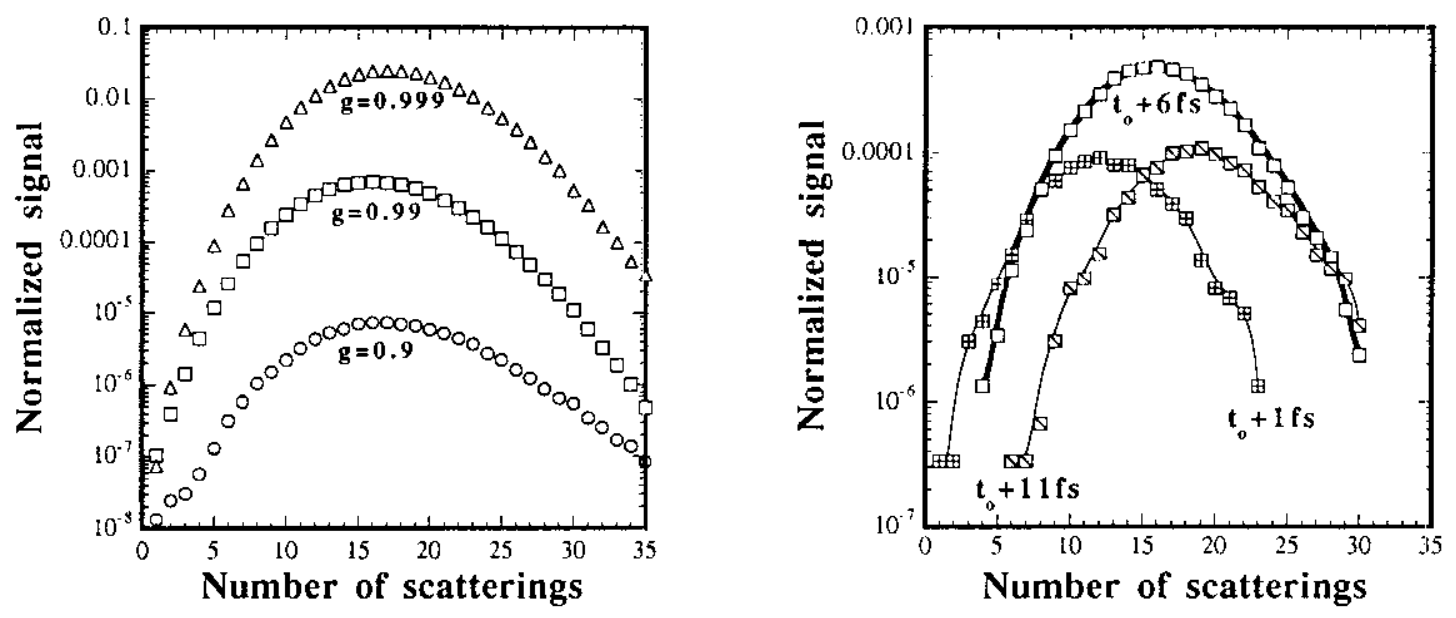

Fig. 6a, b: a) Detected signal normalized to total number of census photons versus number of scatterings and anisotropy $g$ for $\alpha=233 \mathrm{~cm}^{-1}, z_{\mathrm{d}}=800 \mu \mathrm{m}$ and perfectly reflecting boundary condition at $z=400 \mu \mathrm{m} . \mathrm{b}$ ) Detected signal normalized to total number of census photons versus number of scatterings for three times of arrival: $t_{0}+1 \mathrm{fs}, t_{0}+6 \mathrm{fs}, t_{0}+11 \mathrm{fs}$, where $t_{0}$ is time of arrival of an unscattered photon returning from the vessel wall. Parameters same as in a) with $g=0.99$.

$\left(t_{0}+1 \mathrm{fs}\right)$ is still associated with return photons undergoing many small-angle scatterings (12). The bulk of the return signal is detected around $t_{0}+6 \mathrm{fs}$ with the most probable number of scatterings about equal to 16 .

The corresponding path length increase for this delay time is about $1.3 \mu \mathrm{m}$, giving an average scattering angle of nearly $3^{\circ}$ which compares very favorably with $\theta_{0}$ obtained from Eq. (6). This example illustrates the point that an OCT signal dominated by multiply scattered photons is not necessarily degraded as long as the path-length increase remains small compared to a coherence length $\Delta z$.

Next, we consider a more realistic simulation of a vessel geometry and explore the role of noise in OCT. In particular, we add a vessel wall to the above simulations and track all photons which reach the detector regardless of propagation history. For the scattering properties of the vessel wall, we use some measured properties of normal human aortal tissue at a wavelength of $1.32 \mu \mathrm{m}^{10} \mu=2.2 \mathrm{~cm}^{-1}, \alpha=233 \mathrm{~cm}^{-1}$ and $g=0.9$.

The effect of Fresnel reflection at the blood-vessel wall boundary is also included by implementing the following index of refraction mismatch: $n_{\text {bloxt }}=1.33, n_{\text {vessel }}=1.39$. The input scattering parameters for blood are unchanged from above. Because of the finite coherence length of our Gaussian quasi-monochromatic source $(\Delta z=24 \mu \mathrm{m})$, the return signal at $z=0$ will have a distribution of phases with width proportional to $\Delta z$. We convolve the simulated return signal with a Gaussian point-spread function of FWHM $\Delta z / c$ to more realistically simulate an experimental OCT signal. Figure (7a) shows the simulated return signal (per unit length) versus distance to the vessel wall with and without Fresnel reflection. In the case of Fresnel reflection, a large enhancement in signal at the vessel boundary is evident. According to Snell's Law, ${ }^{11}$ the increase in time-integreted signal with Fresnel reflection should be about $0.05 \%$ of the signal for the fully reflecting case [See Fig. (5)], which closely matches the simulation predictions. In a time-resolved sense, the simulated increase in (smoothed) signal at the vessel wall is nearly an order of magnitude larger with Fresnel reflection, compared to a factor-of-five level of enhancement from changes in scattering anisotropy alone, i.e., $\Delta g$. This predominance of Fresnel reflection over anisotropy changes across interfaces is consistent with the conclusions of Reference 2. However, unless the vessel wall is oriented nearly normal to the GRIN lens and specular reflection is dominant, Fresnel reflection is not likely to be an important tool for vascular OCT. 


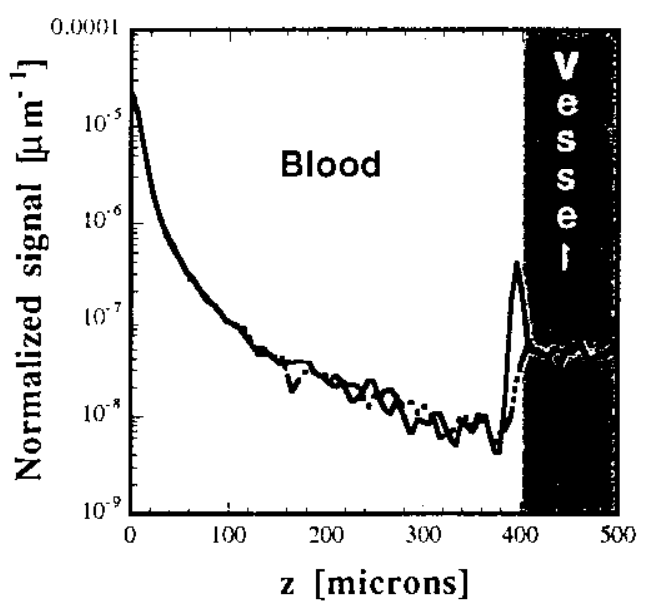

Fig. 7: Normalized detected signal per unit length versus distance for $g=0.99$ (blood), $g=0.9$ (vessel) with Fresnel reflection (solid line) and without (dashed line). Integrated signal reception is 0.00026 of incident signal of which $3 \%$ is from vessel wall.

\section{LATIS Monte Carlo simulations: Mie scattering}

So far we have concentrated on the Henyey-Greenstein formalism for describing anisotropic scattering, both analytically [Section II] and computationally [Section III]. A useful next step is to implement the theory of Mie scattering ${ }^{12}$ in the Monte Carlo package of LATIS. Mie scattering provides three important advantages over Henyey-Greenstein scattering: (1) improved accuracy, (2) inclusion of polarization elfects, and (3) the capability for modeling anisotropic media. This Section looks primarily at the first area with particular emphasis on the details of the angular dependence of scattering.

The theory of Mie scattering is based on solving Maxwell's equations for the fields inside and outside the scattering body and applying appropriate matching boundary conditions. For a spherical scatterer, the wavelength of incident light $\lambda$, radius of scatterer $r_{0}$, and indices of refraction are required to determine the solution. In this way, an amplitude of scattered radiation versus angle can be obtained from first principles. Here, we first compare the Henyey-Greenstein and Mie phase functions by adopting spherical scatterers in
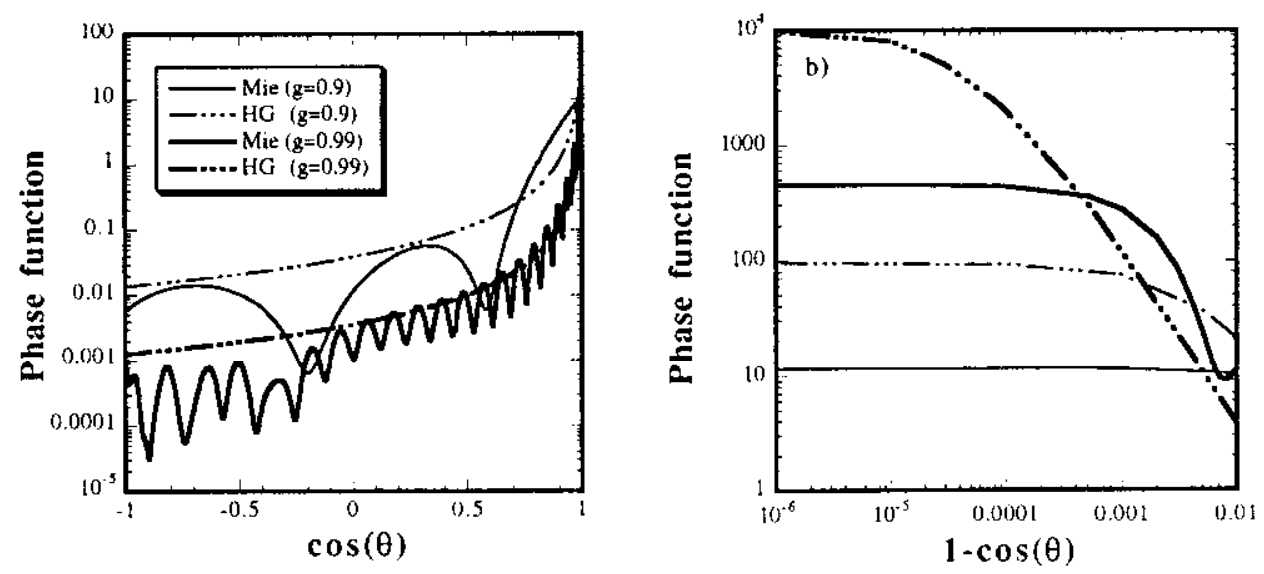

Fig. 8a-b: a) Mie and Henyey-Greenstein phase functions versus $\cos (\theta)$ for $g=0.9,0.99$. b) Enlargement of a) near $\cos (\theta)=1$. 

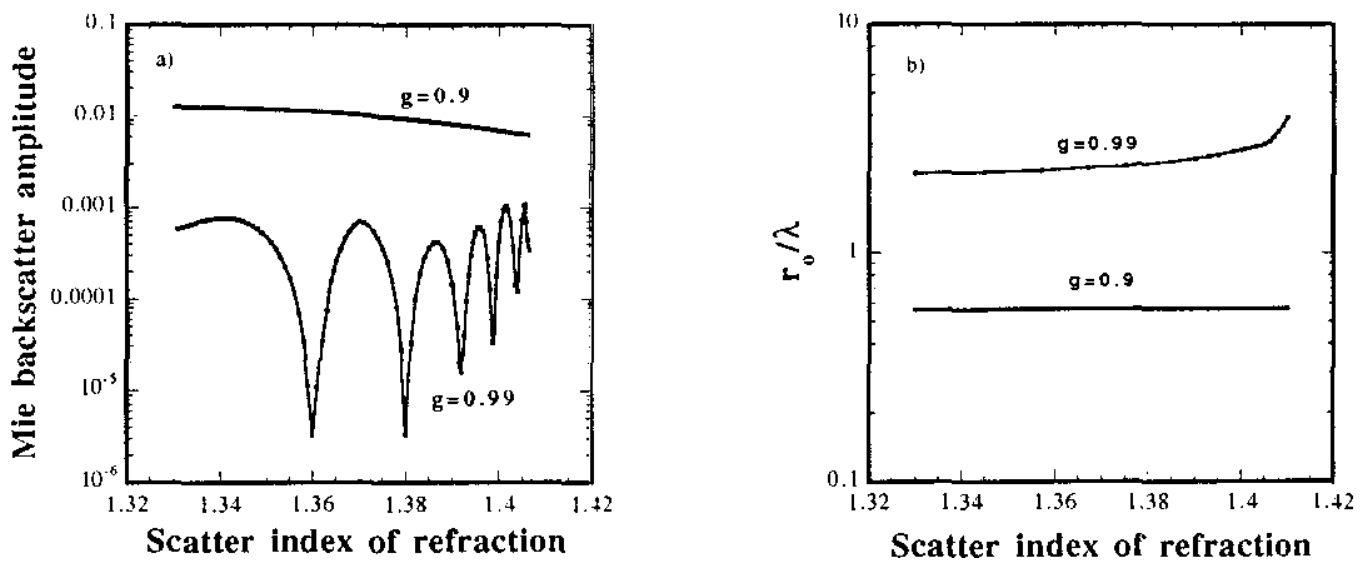

Fig. 9a-b: a) Backscatter amplitude for Mie phase function versus index of refraction of scattering medium for $g=0.9,0.99$. Index of refraction of background medium is taken to be 1.33. b) Ratio of scattering particle radius to wavelength $r_{\mathrm{o}} / \lambda$ vs scatter index of refraction.

the Mie theory and adjusting $r_{\mathrm{o}} / \lambda$ to match $\left\langle\cos (\theta)>_{\text {Mie }}\right.$ with $g$. Figures $(8 \mathrm{a}-\mathrm{b})$ shows a comparison of the two types of phase functions for $g=0.9,0.99$. For both values of anisotropy, the index of refraction of the scatterer $n_{\text {scat }}$ is taken as 1.41 and for the background medium, $n_{b}=1.33$. The required $r_{\mathrm{o}} / \lambda$ with this choice of indices of refraction is 0.57 for $g=0.9$, and 3.9 for $g=0.99$. We see from Fig. (8b) that the Mie scattering phase function with its characteristic lobe structure is not as forward peaked as the Henyey-Greenstein phase function and that the backscattering amplitude is about a factor-of-three smaller with Mie scattering. For smaller index of refraction mismatches, the backscatter amplitude can be a particularly sensitive function of the scattering index of refraction as shown in Fig. (9) for the case of $g=0.99$. This sensitivity diminishes for weaker anisotropy or for larger values of $n_{\text {scat }}$. In contrast, peak forward scattering is little affected at low values of $n_{\text {scat }}$ but exhibits more sensitivity as $n_{\text {scat }}$ increases. All of these properties of the Mie phase function and their consequences for OCT can be gauged by repeating the Monte Carlo

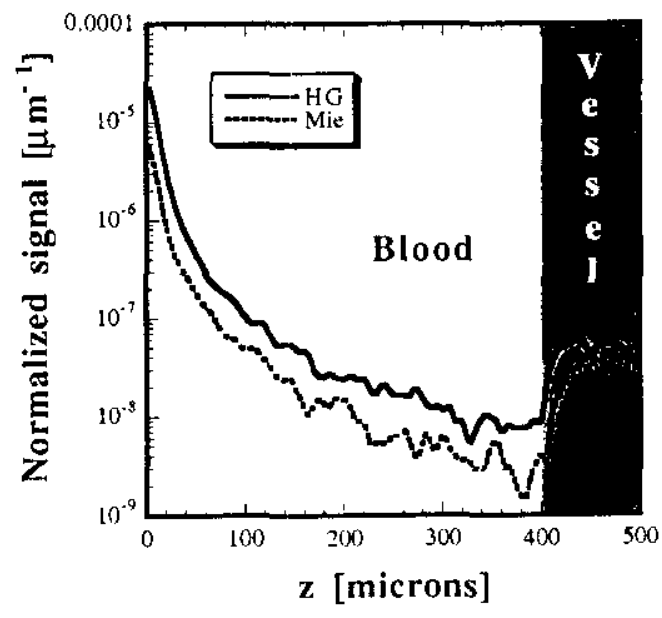

Fig. (10): Normalized detected signal per unit length versus distance for $g=0.99$ (blood), $g=0.9$ (vessel) with no Fresnel reflection for Henyey-Greenstein phase function (solid line) and Mie phase function (dashed line). Integrated signal reception is $0.00024(0.000075)$ of incident signal of which $3 \%(6 \%)$ is from vessel wall for Henyey-Greenstein (Mie) scattering. 
simulations from Section III but using the Mie scattering phase functions depicted in Figs. (8a-b). In Fig. (10) we show a direct comparison of the effect of the Henyey-Greenstein and Mie phase functions on the received signal from a vessel wall at $z=400 \mu \mathrm{m}$ in the absence of Fresnel reflection, cf. Fig. (7). The return signal profiles are similar between the two cases with the Mie phase function responsible only for a $30 \%$ reduction in signal from the vessel wall. However, the signal discrimination near the vessel wall is nearly $50 \%$ larger in the case of Mie scattering. Interestingly, the fraction of photons detected in the two cases differs by a factor-of-three which is very close to the ratio of backscattering amplitudes for the two phase functions, cf. Fig. (8a).

\section{LATIS Monte Carlo simulations: polarization-sensitive OCT}

With Mie scattering now implemented in LATIS, we can start addressing what role polarization can serve in optimizing vascular OCT. In particular, we ask if further signal discrimination can be realized by using a polarized light source and monitoring the degree of polarization versus tissue depth. The degree of polarization of a Monte Carlo photon is described by the Stokes vector $S=\{I, Q, U, V\}$ whose evolution under scattering from a dielectric sphere is governed by the Mueller matrix $M$ :

$$
\left(\begin{array}{l}
I \\
Q \\
U \\
V
\end{array}\right)_{f}=\frac{1}{k^{2} r_{3}^{2}}\left(\begin{array}{ccccc}
M_{11} & M_{12} & 0 & 0 \\
M_{12} & M_{11} & 0 & 0 \\
0 & 0 & M_{33} & M_{34} \\
0 & 0 & -M_{34} & M_{33}
\end{array}\right)\left(\begin{array}{l}
I \\
Q \\
U \\
V
\end{array}\right)_{i}
$$

where $k$ is the wavenumber and $M_{11}{ }^{2}=M_{12}{ }^{2}+M_{33}{ }^{2}+M_{34}{ }^{2}$. The degree of linearly polarized light is described by $Q$ :

$$
Q \equiv \frac{\left.\left\langle\mid E_{x}\right\rangle^{2}\right\rangle-\left\langle\left|E_{y}\right|^{2}\right\rangle}{\left\langle\left|E_{x}\right|^{2}\right\rangle+\left\langle\left|E_{v}\right|^{2}\right\rangle},
$$

where $E_{\mathrm{x}}, E_{\mathrm{y}}$ are the transverse components of the electric field. Linearly polarized $(Q=-1)$ Monte Carlo

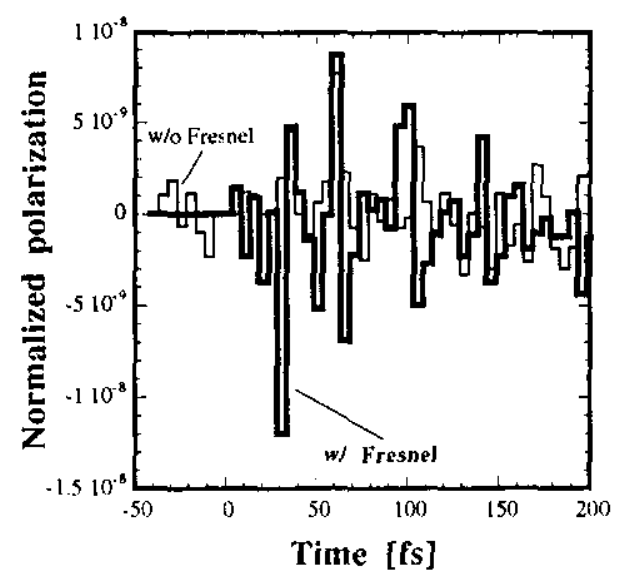

Fig. 11: Normalized $Q$ polarization signal versus time relative to minimum transit time $t_{\mathrm{o}}$ of unscattered photon to vessel wall and back with Fresnel reflection (double solid line) and without (solid). Parameters of simulation are same as in Fig. (9): normalization is relative to total number of launched photons of which 0.000075 return to detector. 
photons are launched from $z=0$ toward the vessel wall and monitored for residual polarization state as a function of pathlength. Figure (11) shows the resulting polarization profile with and without Fresnel reflection. In both cases little, if any, discernible effect of polarization on signal discrimination is evident for this geometry. For the case with Fresnel reflection, a possible $Q<0$ feature is present for the least scattered return photons, but the duration and strength is too meager to be of practical use.

\section{Summary}

We have developed modeling tools to help in our understanding of photon scattering behavior in turbid media for application to OCT. An analytical model has been presented which provides a scaling-law relation for optimizing coherent signal strength. The Monte Carlo photonics in LATIS has been adapted to study scattering phenomena for two choices of phase function: Henyey-Greenstein and Mie. Important differences are found between the two descriptions of scattering, particularly for the backscattering amplitudes. For theparticular case we have studied, the Mie theory of scattering gives reduced return signal but the level of signal discrimination shows some enhancement compared to the Henyey-Greenstein formulation. We have also monitored the photon scattering histories of OCT photons and found that the vast majority of photons returning to the detector have undergone many small-angle forward scatterings. Thus, a useful OCT signal need not be composed of singly scattered photons for utility but may include, if not require, a preponderance of multiply scattered photons. The inclusion of Mie scattering in LATIS makes available the capability to study polarization-sensitive OCT. A preliminary study of polarization effects in an isotropic vessel shows little advantage in using polarized light to date, but further work and extension to anisotropic media are planned.

\section{Acknowledgments}

This work was performed under the auspices of the U.S. Department of Energy by Lawrence Livermore National Laboratory under Contract No. W-7405-Eng-48.

${ }^{\dagger}$ Present address: VISX, Inc., 3400 Central Expressway, Santa Clara, CA 95051

\section{References}

\footnotetext{
${ }^{2}$ Y. Pan, R. Birngruber, J. Rosperich, and R. Engelhardt, Appl. Optics 34 (28), 6564 (1995).

${ }^{3}$ J.M. Schmitt and A. Knüttel, J. Opt. Soc. Am. A 14 (6), 1231 (1997).

${ }^{4}$ J.M. Schmitt, S.H. Xiang, and K.M. Yung, Journal of Biomedical Optics 4 (1), 95-105 (1999).

${ }^{5}$ G. Yao and L. Wang, "Multiple scattering optical coherence tomography signal: Monte Carlo modeling and experimental study", in Coherence Domain Optical Methods in Biomedical Science and Clinical Applications III, Valery V. Tuchin, Joseph A. Izatt, Editors, Proceedings of SPIE Vol. 3598, 10-17 (1999). ${ }^{6}$ R.A. London et al., Appl. Optics 36, 9068 (1997).

${ }^{7}$ R.C. Kirkpatrick et al., "Indirect solar loading of waste heat radiators", Proc. Space 88, published by the American Society of Civil Engineers, S.W. Johnson and J.P. Wetzel, ed. (1988).

${ }^{8}$ L.H. Wang, S.L. Jacques, and L.Q. Zheng, Computer Methods and Programs in Biomedicine 47, 131+146 (1995).

${ }^{10} \mathrm{M}$. Essenpreis, Thermally induced changes in optical properties of biological tissues, University college, London, England (1992).

"J.D. Jackson, Classical Electrodynamics, Wiley \& Sons, New York (1975).

${ }^{12}$ C.F. Bohren and D.R. Huffman, Absorption and scattering of light by small particles, Wiley \& Sons, New York (1983).
} 
